\title{
Learning from failure: congestive heart failure in the postgenomic age
}

\author{
Ivor J. Benjamin'1 and Michael D. Schneider²
}

\begin{abstract}
1Division of Cardiology, Department of Internal Medicine, University of Utah Health Sciences Center, Salt Lake City, Utah, USA ${ }^{2}$ Center for Cardiovascular Development, Departments of Medicine, Molecular \& Cellular Biology, and Molecular Physiology \& Biophysics, Baylor College of Medicine, Houston, Texas, USA.
\end{abstract}

\begin{abstract}
The prognosis of heart failure is worse than that of most cancers, but new therapeutic interventions using stem and other cell-based therapies are succeeding in the fight against it, and old drugs, with new twists, are making a comeback. Genetically engineered animal models are driving insights into the molecular mechanisms that cause hearts to fail, accelerating drug discoveries, and inspiring cell-based therapeutic interventions for both acquired and inheritable cardiac diseases.
\end{abstract}

Heart failure threatens to eclipse infectious disease as the major cause of death worldwide, with the prevalence rising as populations age, Western diets are emulated, and the rate of acute mortality from myocardial infarction subsides (1-3). Given the pace of these 3 trends, recognition of the emerging heart failure epidemic by clinicians, patients, public health officials, the pharmaceutical and biotechnology industries, and policy makers, already long overdue, is crucial for coping with the medical and economic challenges that loom ahead. Thanks to the efforts of scientists worldwide, however, substantial progress is being made toward understanding the underlying etiology, pathogenesis, and mechanisms of heart failure, and, ultimately, toward the prevention of heart failure.

This review series will address selected but complementary and intricately interrelated triggers, such as oxidative stress, nitrosative stress, signal transduction, alterations in intracellular calcium handling, mitochondrial dysfunction, inherited mutations, and myocyte loss, that culminate in heart failure (Figure 1). Emphasis is given, too, to the nodal control points in signal transduction and transcription, whose modulation holds especially salutary promise, and to the nascent field of cardiac regeneration as a therapeutic reality.

\section{Oxygen, oxidative stress, and hypoxia in heart failure}

Aerobic organisms consume molecular oxygen for normal respiration and obligatorily generate toxic byproducts with double-edged beneficial and deleterious consequences. Frank Giordano begins the series with a biochemical overview of oxidative metabolism, a primer on the multiple mechanisms for production, detoxification, and ultimately, modulation of ROS (4). The review concludes with an overview of the transcription factor hypoxia-inducible factor 1 , whose role is well established in oxygen homeostasis, angiogenesis, and even early embryonic development $(5,6)$ but is less certain in chronic heart failure. In contrast to acute ischemia and reperfusion injury, heart failure has only recently been understood as a likely consequence of ongoing oxidative damage: mere markers of oxidative damage do not suffice to establish cause-and-effect relationships in disease pathogenesis, and stronger evidence now has begun

Nonstandard abbreviations used: HDAC, histone deacetylase; PGC-1, PPAR $\gamma$ coactivator-1; PKA, cAMP-dependent protein kinase; RyR, ryanodine receptor; SERCA, sarcoplasmic reticulum $\mathrm{Ca}^{2+}$-ATPase.

Conflict of interest: The authors have declared that no conflict of interest exists.

Citation for this article: J. Clin. Invest. 115:495-499 (2005).

doi:10.1172/JCI200524477. to appear. What pathways best implicate ROS in the pathogenesis of heart failure? By what combination of means does chronic ROS production contribute to myocardial dysfunction and eventual progression to heart failure? In exact biochemical and biophysical terms, how do ROS reduce the calcium sensitivity of myofilaments and impair cardiac contractility?

\section{Nitric oxide in heart failure}

The balance between antioxidant and pro-oxidant systems establishes the redox state in cells. Conventional wisdom holds that oxidative damage of target macromolecules, as a byproduct of ROS, plays a central and direct role in disease pathogenesis. This concept is being challenged by emerging evidence that $S$-nitrosylation of proteins by reactive nitrogen species occurs at specific cysteine residues, with greater selectivity than that of protein oxidation. Additionally, protein oxidation, competitively or allosterically, alters proteins' reactivity to NO. According to this view, modulation of NO-based signaling is the principal consequence and $S$-nitrosylation a primary effector of altered redox states. Joshua Hare and Jonathan Stamler review the key sources of ROS and reactive nitrogen species (e.g., hemoglobin, NO synthase), modes of their induction (e.g., $\beta$-adrenergic signals), spatial compartmentalization, and counter-regulatory inhibitors (e.g., S-nitrosoglutathione reductase) (7). Dysregulation of $S$-nitrosylation is proposed as a mediator of diabetes, atherosclerosis, myocardial infarction, hypertrophy, and ultimately, heart failure. Causal mechanisms have been elegantly worked out in exquisite molecular detail for 2 highly relevant and suggestive physiological processes - excitation-contraction coupling and vascular reactivity, the latter resulting from impairment of erythrocyte-mediated dilatation in the vasculature. Direct evidence is lacking, though, for other suggested effects, warranting future work with judicious use of animal models and parallel studies of the human disorder.

\section{Causative mutations in heart failure}

From the perspective of etiology in heart failure, no recent insight has been greater than the discovery that hypertrophic and dilated cardiomyopathies can result from mutations in genes encoding an astonishingly broad range of cardiac proteins. Hiroyuki Morita, Jonathan Seidman, and Christine Seidman review the spectrum of causative mutations and new insights into their mechanisms of action (8). To date, hundreds of mutations that cause hypertrophic cardiomyopathy have been pinpointed, with 


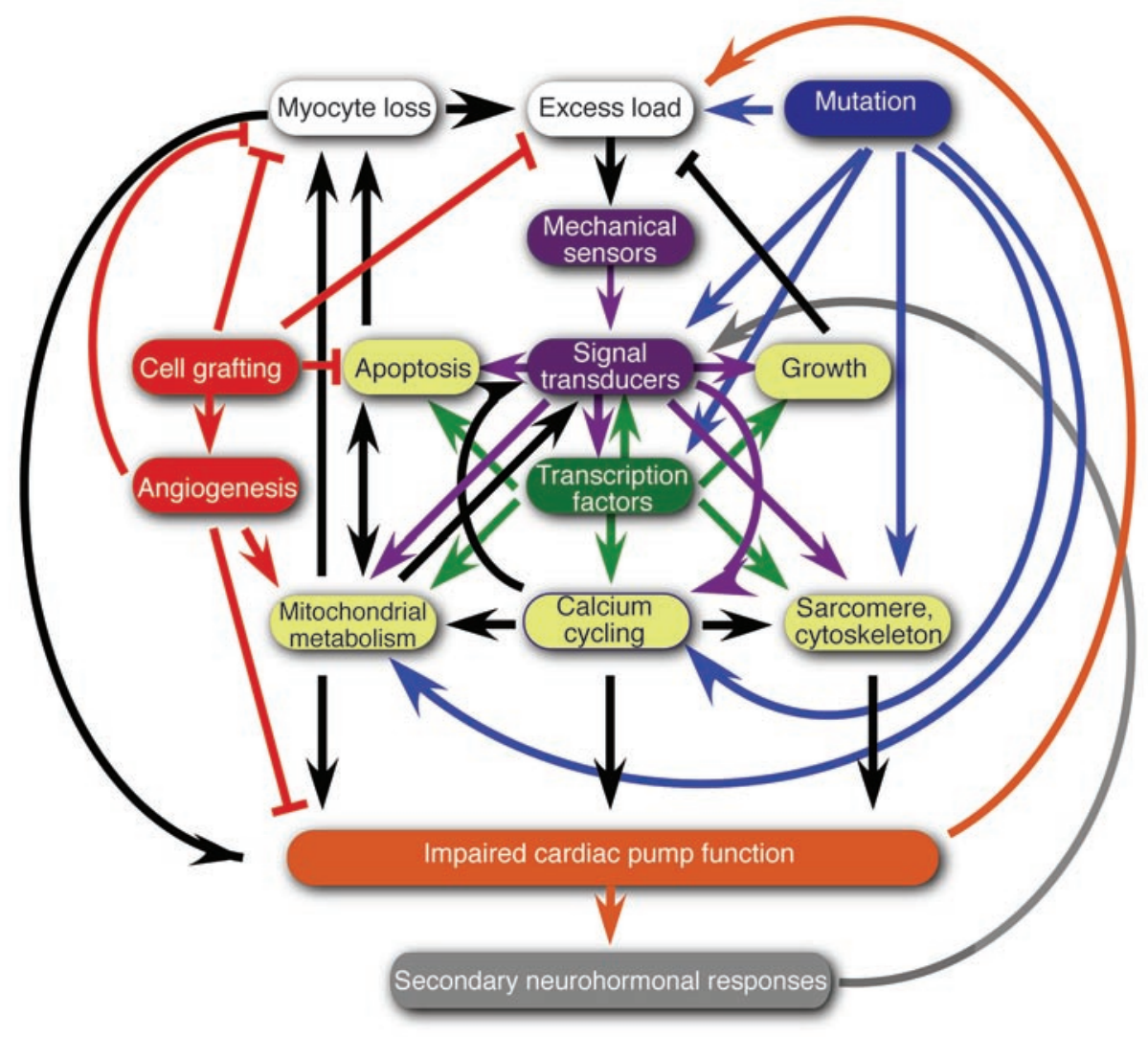

Figure 1

A partial wiring diagram of biological circuits for heart failure. Impaired pump function after myocyte death from myocardial infarction or abnormal loading conditions such as found in hypertension (white) activate a biomechanical stress-dependent signaling cascade (purple). The responsible targets of altered signal transduction cascades in heart failure include transcription factors, coactivators, and corepressors for cardiac gene expression (green) as well as the effector mechanisms like calcium cycling, metabolism, growth, and apoptosis (yellow) that culminate in ventricular dysfunction (orange) and secondary neurohumoral responses (gray) such as adrenergic drive and intramyocardial growth factors (not shown). Inherited mutations for cardiomyopathy (blue) affect proteins at many of these points and are thought to engage a similar cascade of events in order to elicit the full myopathic phenotype. Cell-based therapies (red), although often envisioned working chiefly or wholly by replacing dead myocytes, probably improve ventricular performance through a combination of mechanisms, including angiogenesis, paracrine signals for myocyte protection, and conceivably augmenting host self-repair.

varying phenotypes from missense mutations in a given protein. An intriguing but disturbing handful of mutations compel a markedly accelerated course. Determining the way in which human myosin heavy chain mutations alter force-generation in single-molecule motility studies is an unquestionable triumph of reductionist research. In contrast, we do not yet comprehend how hyperdynamic properties at this level of organization "trickle up" to myofiber disarray, sporadic cell death, and reactive fibrosis at the tissue level, which illustrates a critical gap in the present understanding of pathogenesis, as opposed to etiology. From one point of view, therefore, many hereditary cardiomyopathies differ little from garden-variety acquired cardiac disorders; the instigating signal is known in both cases, whether it is a mutation, long-standing hypertension, or past ischemic injury, yet the workings of disease progression are still cryptic. Because genetic defects alter cells in highly precise and defined ways, it is reasonable to hope that dissecting their effector pathways might prove simpler than dissecting those of other forms of heart disease. The authors remind us that human mutations are an experiment of nature through which extraordinary details of cardiac proteins' structure and function have come to be unmasked - not only for the sarcomeric and cytoskeletal proteins that are most familiar in hereditary heart disease, but also for genetic defects in energy-generating mechanisms, calcium-cycling mechanisms, and transcriptional control.

\section{Protein kinases in heart failure} Viewing heart failure as a disorder of cell signaling - determining the way in which inciting cues are coupled to their effectors - has come to occupy center stage for much of the most innovative work on heart failure pathogenesis and its inverse, therapeutic opportunity, with protein kinases and phosphatases seen as having especially cogent roles. In considering the labyrinthine maze of signal transduction events leading up to heart failure, Gerald Dorn and Thomas Force focus chiefly on 2 central aspects of protein kinase signaling: the $G_{\alpha q} / G_{\alpha 11} /$ protein kinase $\mathrm{C}$ and phosphoinositide $3^{\prime}$ kinase/Akt pathways (9). Clearly, not all cardiac growth is equivalent. What biochemical mechanisms distinguish normal postnatal cardiac growth (or, for that matter, athletic conditioning) from the enlargement triggered by workload in disease states or by other pathobiological stress? Apart from the existence of many potentially pivotal or contributory kinases operating in series or in parallel, a further challenge is the sheer complexity of several kinase families taken individually. Where a panoply of closely related kinases coexist, as is the case for protein kinase $\mathrm{C}$, do the various family members serve redundant or recognizably divergent functions, and what genetic models offer the best chance for an unambiguous answer? The functional and spatial compartmentalization of signaling molecules in heart failure are being scrutinized. How does kinase translocation in cardiac muscle change in cardiac disease? How does localization influence activity and target, and might this property be exploited clinically, redirecting kinases away from sites of pathogenic activation or effect? What signals converge on a nodal control like Akt, and what effector pathways operate downstream? If other protein kinases, such as glycogen synthase kinase-3 $\beta$, act to suppress cardiomyocyte hypertrophy in culture, could this hold true in vivo too? What are the other critical targets, and might suppression of hypertrophy by such means prove to be therapeutically fruitful in humans? 


\section{Transcriptional reprogramming in heart failure}

Besides the direct effects of signaling cascades on excitation-contraction coupling, the contractile apparatus, energy production, and myocyte survival itself, many if not all of the signal transduction mechanisms that are engaged in heart failure also converge on the cell nucleus, with long-term effects on virtually every aspect of cardiac function through the enforcement of altered gene expression. Transcriptional reprogramming has moved beyond being viewed as just a molecular marker of hypertrophic phenotypes or as an ostensibly beneficial adaptation to being considered instrumental to pathogenesis in failing hearts and, hence, a logical target for therapeutic interventions. Timothy McKinsey and Eric Olson describe progress in the understanding and modulation of transcription factors and chromatin-remodeling enzymes that execute the hypertrophic gene program during pathological remodeling of the heart (10). Pathways that impinge on nuclear-cytoplasmic shuttling by the nuclear factor of activated T cells (a coactivator for GATA transcription factors) and class II histone deacetylases (HDACs) (corepressors for myocyte enhancer factor-2) have proven to be especially fruitful in this regard. For example, stress-mediated nuclear export of class II HDACs - triggered by calmodulin-dependent protein kinases and by a protein kinase $\mathrm{C} /$ protein kinase $\mathrm{D}$ cascade - permits myocyte enhancer factor-2 to recruit histone acetylases, remodel repressed chromatin to an open state, and activate hypertrophy-associated genes. Counterintuitive phenotypes have resulted in some instances of manipulating nominally pro- or antihypertrophic pathways. Here, paradoxical results, contrary in direction to the expected ones, are possibly related to coexisting class I and class II HDACs. Despite this recent complexity, the logic of viewing heart failure as a transcriptional disorder is unusually sound, and the pace of discovery is accelerating to an almost unimagined degree through the implementation of high-throughput screening technologies.

\section{Mitochondrial energy metabolism in heart failure}

Recent years have witnessed an explosion in our understanding of the metabolic pathways, energy substrate utilization, and sensing mechanisms essential for mitochondrial biogenesis and ATP generation in both the normal and failing heart. Together, biochemical and genetic lines of evidence suggest that the normal transition from glucose oxidation during fetal development to fatty acid oxidation in the adult heart reverses during certain disease states (e.g., hypertrophy, ischemia) or becomes exaggerated in others (e.g., uncontrolled diabetes). Janice Huss and Daniel Kelly summarize the major transcriptional network implicated in mitochondrial biogenesis and function (11), encompassing PPAR $\gamma$ coactivator-1 (PGC-1), the ensemble of downstream nuclear receptor partners (e.g., PPARs and estrogen-related receptors), converging molecular signals (e.g., $\mathrm{Ca}^{2+}, \mathrm{NO}, \mathrm{MAPKs}$, $\beta$-adrenergic mechanisms, cAMP), and signal transduction pathways (e.g., p38 MAPK and ERKs). How are signals upstream of PGC-1 $\alpha$ sensed and integrated? What are the molecular biosignatures of the failing heart? What is the evidence that a shift to glucose utilization from fatty acid oxidation is adaptive or maladaptive? How do altered mitochondrial energetics contribute to heart failure? Diabetes has also emerged as a major public health problem with widespread cardiovascular ramifications, although the vasculature as a target has received far greater attention than the cardiac myocyte. Interestingly, though, chronic upregulation of PGC- $1 \alpha$ in myocardium leads to ventricular dysfunction, a phenotype mimicking the diabetic state at the cellular and ultrastructural levels. Important strides have been made regarding the ways in which high circulating fatty acids and increased insulin resistance in myocytes contribute to increased fatty acid utilization in diabetic states. New means to reverse these metabolic abnormalities herald opportunities for therapeutic intervention, perhaps targeted to PGC- $1 \alpha$-dependent control of oxidative energetics and of lipid metabolism in the failing heart.

\section{Intracellular $\mathrm{Ca}^{2+}$ handling in heart failure}

Abnormal intracellular calcium handling, a hallmark of cardiac contractile abnormalities, has been attributed to several major $\mathrm{Ca}^{2+}$ regulatory proteins of the sarcoplasmic reticulum: namely, the calcium-release channel (ryanodine receptor [RyR]), sarcoplasmic reticulum $\mathrm{Ca}^{2+}$-ATPase (SERCA2), which mediates $\mathrm{Ca}^{2+}$ uptake into the sarcoplasmic reticulum, and phospholamban, whose inhibition of SERCA2 is alleviated by cAMP-dependent protein kinase (PKA). Whereas $\beta$-receptor blockade of excess sympathetic nervous system drive and inhibition of the renin-angiotensin system have become standard therapies for heart failure, only recently have their mechanisms of action been defined elegantly in molecular terms. Because alterations in $\mathrm{Ca}^{2+}$ cycling often precede overt heart failure, recent intense interest has focused on the key $\mathrm{Ca}^{2+}$ regulatory proteins as potential therapeutic targets. Masafumi Yano, Yasuhiro Ikeda, and Masunori Matsuzaki summarize the molecular events governing $\mathrm{Ca}^{2+}$ release and uptake, the recent characterization of clinical mutations in these proteins associated with arrhythmogenesis or ventricular dysfunction, and controversies related to the major mechanisms by which phosphorylation of RyR by PKA regulates $\mathrm{Ca}^{2+}$ handling, a process involving FKBP12.6 dissociation from the RyR macromolecular complex (12). SERCA2-dependent effects on cardiac contractility and $\mathrm{Ca}^{2+}$ handling in the heart also modulate many complex events that control the channel-gating property of RyR. The angiotensin II-receptor blocker valsartan abrogates catecholamineinduced hyperphosphorylation of RyR2 by PKA and subsequently inhibits the adverse $\mathrm{Ca}^{2+}$ leak associated with heart failure. More directly aimed at calcium handling itself, an intriguing hypothesis explored in this review is the stabilization of conformational changes of the RyR macromolecular complex using small molecules, thereby reversing the deleterious effects of altered phosphorylation. Similar opportunities for targeted therapies herald likely advances in our armamentarium against heart failure in humans.

\section{Apoptosis in heart failure}

Among the most striking conceptual revisions in recent heart failure research is the appreciation of ongoing, sporadic myocyte death in failing human myocardium and in animal models, through which a causal role for apoptosis has been proven in ventricular dysfunction and the responsible circuitry defined. Roger Foo, Kartik Mani, and Richard Kitsis summarize what is known about the highly conserved machinery for apoptotic signaling via cell surface death receptors and the mitochondrion - the extrinsic and intrinsic pathways, respectively - and their respective means of activating the specific proteases (caspases) that are largely responsible for apoptotic demise (13). Beyond just these two classical inputs and the classical role of caspase-mediated cleavage in bringing about cell death, the authors point to novel sensors for apoptosis in heart failure, which involve the endoplasmic reticulum or telomere uncapping, as well as to sublethal caspase activation in failing myocardium as a novel way for altered protein and cell function to arise. The dysregulation of death and survival pathways in failing hearts, together with accumulating proof that cardiac myocyte apoptosis is a causal element not merely in acute ischemic injury but also in more chronic heart failure states, 
poses a challenge: can cell death be intercepted therapeutically, and, if so, what are the long-term consequences on ventricular remodeling and function? In contrast to suppressing apoptosis acutely, sustained interference with the machinery for programmed cell death might run afoul of tumor surveillance mechanisms that selectively eliminate mutant cells from the body. Consequently, there is keen interest in interventions that build specifically on those few components of apoptosis that are heart-specific or -enriched.

\section{Cell therapy for cardiac repair}

In the circumstance of devastating, acute muscle cell death from myocardial infarction - and perhaps in apoptotic cardiomyopathies as well - merely enhancing myocyte performance and averting adverse adaptations may not suffice to correct the underlying biology (insufficient muscle cell replacement by endogenous repair mechanisms to offset the extent of muscle cell loss). The prospect of cardiac repair as a problem in regeneration is intriguing, novel, and complex, and the rapidity with which human trials have progressed is a remarkable example of bench-to-bedside translation. Stefanie Dimmeler, Andreas Zeiher, and Michael Schneider summarize the major published human trials in this field, highlight the resulting key uncertainties, and interpret the basic research findings that are paving the way for the next generation of clinical studies (14). How might cell mobilization, homing, and migration best be enhanced? To what extent do grafted cells function as replacements for cardiac muscle, and to what extent do their benefits depend on different mechanisms altogether, such as angiogenesis or the production of secreted cell-survival factors $(15,16)$ ? What is the evidence for and against transdifferentiation of bone marrow-derived cells into cardiac muscle, and is all bone marrow equivalent for this purpose? If cardiac muscle itself contains resident progenitor or stem cells, as was previously cryptic but now appears to be the case, what are these cells' properties, and what advantages, if any, distinguish them from the cells in current clinical use? What specific genes mediate the capacity of heart-derived progenitor cells to form daughter cardiac myocytes so efficiently? What extracellular cues provide the biological signals for cardiac neomyogenesis in the adult? With the answer presumably dependent on type, what functional properties of various donor cells are well-posed targets for pharmacological or even genetic enhancements?

\section{Caveat lector (let the reader beware)}

The series of reviews presented in this issue of the JCI provides both a synoptic view and an insightful critique of where we are, and are not, in contemporary, postgenomic, heart failure research. No article, series, or series of series could cover the field in its entirety, however, and several admonitions are noteworthy.

First, many but not all of the insights gleaned from genetically engineered mouse models are accurate replications of human heart failure, and the counterexamples underscore one of the central experimental challenges and opportunities in this burgeoning field. Calcium cycling is arguably the aspect of human myocardium least faithfully represented in the mouse and other genetically tractable model organisms. The resting heart rate of 500 or more in normal mice highlights the underlying physiological discrepancy, which is accompanied by corresponding disparities in several key features of ion homeostasis; for instance, loss of phospholamban enhances cardiac function in mice but causes lethal dilated cardiomyopathy in humans (17). What would be the effect in patients of interfering with this protein? In short, when are genetically engineered animal models at most risk to mislead as surrogates for human heart failure? Where pathophysiological relevance of mouse models to humans is in question, what alternatives, such as viral gene delivery to larger mammals, might best be pursued to sort out gene function?

Conversely, not even large mammal studies of cardiac repair using skeletal myoblasts discerned an increased hazard of arrhythmias until that risk became apparent clinically. Many features of the clinical substratum in heart failure would be cumbersome to reproduce or unworkable (aging, diffuse and distal coronary artery disease, and metabolic syndrome, among them). Even the species that is anatomically fittest as a model will surely fall short on some predictions. Balancing the need for proof of safety and efficacy against the inherent uncertainty of all tests short of the clinical trial requires discernment and nuanced thinking on the part of scientists, physicians, and regulatory agencies alike.

Finally, the propulsive growth of genetics applied to disorders of the myocardium has to date yielded clues chiefly or solely in the context of deterministic human single-gene disorders and, in mice, to engineered mutations of single-candidate genes. It is plausible, although as yet unproven, that heart failure will surrender at least some of its secrets if scrutinized from the perspective of complex, multigenic inheritance. Suggestions that this might be so are found in exploratory studies of modifier and quantitative trait loci that influence survival and ventricular function in mice with dilated cardiomyopathy (18). New genomic resources for the genetic dissection of complex traits include chromosome substitution strains, each containing a specified chromosome from a donor strain substituted into the recipient host background (19); these and other tools for the analysis of complex traits (20) will unquestionably be put to service in providing a genetic basis for the known variations among mouse strains in cardiac mass, ventricular cavity dimensions, and contractile performance (21). Apart from those obtained by capitalizing more fully on the information in these spontaneous variations, genetically unbiased discoveries are also to be expected from the genome-wide creation of chemical and insertional mutations in mice $(22,23)$, strategies heretofore known better for their success in flies and fish $(24,25)$. Furthermore, advances in the enabling technology for higher-throughput gene association studies, such as genotyping pooled DNA (26) and highly multiplexed genotyping on DNA microarrays (27), offer unprecedented ease and efficiency in the full exploitation of human genetic diversity. Among other strategies, initiatives using multiethnic population samples - encompassing profound disparities in susceptibility or responses to treatment $(28,29)$ - might afford unprecedented opportunities to decode the genetic and genomic underpinnings of human heart failure $(3,30)$.

\section{Acknowledgments}

I.J. Benjamin is supported by the NIH and the Christi T. Smith Professorship Foundation. M.D. Schneider is supported by the NIH, the M.D. Anderson Foundation professorship, the Donald W. Reynolds Foundation, and the Fondation Leducq.

Address correspondence to: Ivor J. Benjamin, Division of Cardiology, Department of Internal Medicine, University of Utah Health Sciences Center, Room 4A100, 30 North 1900 East, Salt Lake City, Utah 84132, USA. Phone: (801) 581-7715; Fax: (801) 581-7735; E-mail: ivor.benjamin@hsc.utah.edu. Or to: Michael D. Schneider, Center for Cardiovascular Development, Baylor College of Medicine, One Baylor Plaza, Room 506D, Houston, Texas 77030, USA. Phone: (713) 798-6683; Fax: (713) 798-7437; E-mail: michaels@bcm.tmc.edu. 
1. Jessup, M., and Brozena, S. 2003. Heart failure. N. Engl. J. Med. 348:2007-2018.

2. Bleumink, G.S., et al. 2004. Quantifying the heart failure epidemic: prevalence, incidence rate, lifetime risk and prognosis of heart failure: The Rotterdam Study. Eur. Heart J. 25:1614-1619.

3. Liew, C.C., and Dzau, V.J. 2004. Molecular genetics and genomics of heart failure. Nat. Rev. Genet. 5:811-825.

4. Giordano, F.J. 2005. Oxygen, oxidative stress, hypoxia, and heart failure. J. Clin. Invest. 115:500-508. doi:10.1172/JCI200524408.

5. Giaccia, A.J., Simon, M.C., and Johnson, R. 2004. The biology of hypoxia: the role of oxygen sensing in development, normal function, and disease. Genes Dev. 18:2183-2194.

6. Ramirez-Bergeron, D.L., et al. 2004. Hypoxia affects mesoderm and enhances hemangioblast specification during early development. Development. 131:4623-4634.

7. Hare, J.M., and Stamler, J.S. 2005. NO/redox disequilibrium in the failing heart and cardiovascular system. J. Clin. Invest. 115:509-517. doi:10.1172/ JCI200524459.

8. Morita, H., Seidman, J., and Seidman, C.E. 2005. Genetic causes of human heart failure. J. Clin. Invest. 115:518-526. doi:10.1172/JCI200524351.

9. Dorn, G.W., II, and Force, T. 2005. Protein kinase cascades in the regulation of cardiac hypertrophy. J. Clin. Invest. 115:527-537. doi:10.1172/ JCI200524178.

10. McKinsey, T.A., and Olson, E.N. 2005. Toward transcriptional therapies for the failing heart: chemical screens to modulate genes. J. Clin. Invest. 115:538-546. doi:10.1172/JCI200524144.

11. Huss, J.M., and Kelly, D.P. 2005. Mitochondrial energy metabolism in heart failure: a question of balance. J. Clin. Invest. 115:547-555. doi:10.1172/ JCI200524405.

12. Yano, M., Ikeda, Y., and Matsuzaki, M. 2005 Altered intracellular $\mathrm{Ca}^{2+}$ handling in heart failure. J. Clin. Invest. 115:556-564. doi:10.1172/ JCI200524159.

13. Foo, R.S.-Y., Mani, K., and Kitsis, R.N. 2005. Death begets failure in the heart. J. Clin. Invest. 115:565-571. doi:10.1172/JCI200524569.

14. Dimmeler, S., Zeiher, A.M., and Schneider, M.D. 2005. Unchain my heart: the scientific foundations of cardiac repair. J. Clin. Invest. 115:572-583. doi:10.1172/JCI200524283.

15. Bock-Marquette, I., et al. 2004. Thymosin beta4 activates integrin-linked kinase and promotes cardiac cell migration, survival, and cardiac repair. Nature. 432:466-472.

16. Schneider, M.D. 2004. Regenerative medicine: Prometheus unbound. Nature. 432:451-453.

17. Haghighi, K., et al. 2003. Human phospholamban null results in lethal dilated cardiomyopathy revealing a critical difference between mouse and human. J. Clin. Invest. 111:869-876. doi:10.1172/ JCI200317892.

18. Le Corvoisier, P., Park, H.Y., Carlson, K.M., Marchuk, D.A., and Rockman, H.A. 2003. Multiple quantitative trait loci modify the heart failure phenotype in murine cardiomyopathy. Hum. Mol. Genet. 12:3097-3107.

19. Singer, J.B., et al. 2004. Genetic dissection of complex traits with chromosome substitution strains of mice. Science. 304:445-448.

20. Churchill, G.A., et al. 2004. The collaborative cross, a community resource for the genetic analysis of complex traits. Nat. Genet. 36:1133-1137.
21. Hoit, B.D., et al. 2002. Naturally occurring variation in cardiovascular traits among inbred mouse strains. Genomics. 79:679-685.

22. Clark, A.T., et al. 2004. Implementing large-scale enu mutagenesis screens in North America. Genetica. 122:51-64.

23. Skarnes, W.C., et al. 2004. A public gene trap resource for mouse functional genomics. Nat. Genet. 36:543-544.

24. Kim, Y.O., Park, S.J., Balaban, R.S., Nirenberg, M., and Kim, Y. 2004. A functional genomic screen for cardiogenic genes using RNA interference in developing drosophila embryos. Proc. Natl. Acad. Sci. U. S. A. 101:159-164.

25. Xu, X., et al. 2002. Cardiomyopathy in zebrafish due to mutation in an alternatively spliced exon of titin. Nat. Genet. 30:205-209.

26. Hinds, D.A., et al. 2004. Application of pooled genotyping to scan candidate regions for association with HDL cholesterol levels. Hum. Genomics. 1:421-434

27. Shapero, M.H., et al. 2004. Mara: a novel approach for highly multiplexed locus-specific snp genotyping using high-density DNA oligonucleotide arrays. Nucleic Acids Res. 32:e181.

28. Taylor, A.L., et al. 2004. Combination of isosorbide dinitrate and hydralazine in blacks with heart failure. N. Engl. J. Med. 351:2049-2057.

29. Small, K.M., et al. 2004. Polymorphisms of cardiac presynaptic alpha2c adrenergic receptors: diverse intragenic variability with haplotype-specific functional effects. Proc. Natl. Acad. Sci. U. S. A. 101:13020-13025.

30. Liggett, S.B. 2004. Genetically modified mouse models for pharmacogenomic research. Nat. Rev. Genet. 5:657-663. 